\title{
Diethylstilbestrol for the treatment of patients with castration-resistant prostate cancer: Retrospective analysis of a single institution experience
}

\author{
TAL GRENADER ${ }^{*}$, YEVGENI PLOTKIN* , MAYA GIPS, NATHAN CHERNY and ALBERTO GABIZON
}

Department of Oncology, Shaare Zedek Medical Center, and Hebrew University-School of Medicine, Jerusalem, Israel

Received August 5, 2013; Accepted September 30, 2013

DOI: $10.3892 /$ or.2013.2852

\begin{abstract}
The aim of the present retrospective study was to evaluate the efficacy and safety of diethylstilbestrol (DES) as treatment for patients with castration-resistant prostate cancer (CRPC) and to identify predicting factors of response to DES. Patients treated with DES during the castration-resistant phase following the failure of prior treatment with LH-RH analogs during the castration-sensitive phase were retrieved from a prostate cancer database of our institution. Patients were treated with a daily dose of DES of 1-4 mg (mean, $2.6 \mathrm{mg}$ ) and anticoagulants for thromboembolic prophylaxis until disease progression. We analyzed their medical records, biochemical prostate-specific antigen (PSA) response and time to disease progression (TDP). Disease response and progression were identified according to the PCWG2 criteria. Patient data were examined using Kaplan-Meier survival analysis and statistical correlation tests with intra-patient comparison of the LH-RH and DES treatment phases. Fortythree DES-treated CRPC patients were found in our database through July 2011. The median age was 66 years. Sixty-three percent of the patients achieved $a \geq 50 \%$ decline in their serum PSA levels during DES therapy. Median TDP was 20.4 months for LH-RH analog treatment in the castration-sensitive phase, and 7.1 months for DES treatment in the castration-resistant
\end{abstract}

Correspondence to: Professor Alberto Gabizon, Shaare Zedek Medical Center, Oncology Institute, P.O. Box 3235, Jerusalem 91031 , Israel

E-mail: alberto.gabizon@gmail.com

*Contributed equally

Abbreviations: DES, diethylstilbestrol; LH-RH, luteinizing hormonereleasing hormone; CRPC, castration-resistant prostate cancer; PSA, prostate-specific antigen; TDP, time to disease progression; SHBG, sex hormone binding globulin, VTE, venous thromboembolism; PE, pulmonary embolism; DVT, deep venous thromboembolism

Key words: diethylstilbestrol, castration-resistant prostate cancer, luteinizing hormone-releasing hormone, prostate-specific antigen phase. Durable responses ( $>1$ year) were observed in $31 \%$ of the patients. Median overall survival was 57 months from the start of the DES therapy. There was no significant correlation between the TDP under LH-RH analogs and under DES therapy among the 38 patients eligible for correlation analysis. However, the magnitudes of serum PSA responses under DES and LH-RH analogs were significantly correlated with each other, and with the TDP under DES therapy. There were no treatment-related deaths. Four patients (9\%) developed thromboembolic complications while under treatment, some of which appeared to be related to a discontinuation of thromboprophylaxis. In conclusion, DES confers substantial clinical benefit in the treatment of CRPC, with a relatively good safety profile when administered with thromboprophylaxis. The use of DES may be effective in CRPC, irrespective of the length of the hormone-sensitive period with LH-RH treatment. The magnitude of PSA response to previous treatment with LH-RH analogs, as well as to DES, was predictive of the duration of response to DES.

\section{Introduction}

Cancer of the prostate gland is the most common malignancy in men (1). Despite advances in diagnosis and treatment of early stages of prostate cancer, the majority of men who suffer from disease recurrence have no potentially curative treatment option (2).

The accepted first-line treatment for advanced prostate cancer is surgical or chemical castration, using LH-RH partial agonists or antagonists or a combination of an LH-RH analog with an anti-androgen (3). With this treatment, $~ 80 \%$ of men experience symptomatic improvement and a reduction in prostate-specific antigen (PSA) serum levels.

After a median time to disease progression (TDP) of $\sim 18$ months (4), all patients ultimately become refractory to hormone therapy as the disease progresses, and these patients have a life expectancy of $\sim 12$ months (5). Once patients become castration-resistant, docetaxel-based chemotherapy was, until recently, the only viable treatment option offering improved survival $(5,6)$. Currently, two new compounds have been approved for hormonal therapy in castration-resistant prostate cancer (CRPC): abiraterone acetate and MDV3100 (enzalutamide). Indeed, CRPC is still amenable to response 
with certain hormonal manipulations. As a result, CRPC does not imply hormone-refractory disease, a term that was used in the past for this condition.

For many years, metastatic CRPC was considered to confer a dismal prognosis; however, the advent of new agents has improved both the prognosis and quality of life for men with this illness.

Until recent years, the prevailing view was that during the course of prostate cancer, new clones of cells are constantly emerging, some of which cease to be androgen-dependent and present with tumor androgen-independent growth. Recently, de novo intratumoral androgen synthesis during progression of prostate cancer has been detected $(7,8)$. Together with androgens produced by the adrenal glands, this intratumoral de novo synthesis is presumed to maintain sufficient intracellular androgen levels for activating androgen receptor target genes. Thus, the tumor is able to grow in spite of relatively low androgen serum levels.

Diethylstilbestrol (DES) is a powerful synthetic estrogen. In the $1940 \mathrm{~s}$, it became the first hormonal treatment shown to inhibit prostate cancer (9-11). Following this discovery, the accepted treatments for advanced prostate cancer became orchiectomy and/or DES for the next two decades (11-13). The Veterans Administration Cooperative Urological Research Group (VACURG) found that DES increased cardiovascular and thrombogenic toxicity at the 5-mg dose. A slight trend for reduced efficacy at the 1-mg dose, led to the use of $3 \mathrm{mg}$ as the standard dose in patients with metastatic prostate cancer $(11,12)$. Low doses $(1-3 \mathrm{mg} /$ day) of DES had fewer cardiovascular side-effects when compared to higher doses. Typically, thromboembolic events occur in $\sim 5 \%$ of patients $(12,13)$.

The discovery of LH-RH analogs, proven to have similar efficacy to that of orchiectomy and DES as first-line therapy, but without increased cardiovascular and thromboembolic toxicity, led to a loss of interest in DES which fell out of favor and stopped being used in most countries in the 1980s (11). Soon afterward, the pharmaceutical production of DES was discontinued, particularly given its high premium insurance cost due to its teratogenicity when used to support pregnancy in the 1950s.

Despite having been researched for an extended period of time, the method by which DES induces a clinical response in CRPC is still unclear. DES has been shown to have a high affinity for binding to androgen receptors, which play a significant role as growth-drivers of prostate cancer (13). Other suggested mechanisms include inhibition of the secretion of gonadotropins and a sharp reduction in free testosterone levels (14). DES also increases the production of sex hormonebinding globulin (SHBG) and prolactin, thus, decreasing the amount of bio-available testosterone $(6,15)$. There is also evidence that estrogens may inhibit synthesis of androgens by tumor tissue, such as reports of estrogens suppressing the levels of tumor testosterone and dihydrotestosterone in an estrogen receptor-independent manner (16).

In 2000, we reported preliminary observations on the activity of DES in CRPC patients treated at Hadassah Medical Center (17). Our aim here was to extend those observations to a larger group of patients treated in our present institution (Shaare Zedek Medical Center) and to investigate factors that may be predictive for the response to DES to aid in the treatment decision-making process.

\section{Materials and methods}

Study population. Patients who received DES were retrospectively retrieved from a database of patients treated for prostate cancer at the Shaare Zedek Medical Center. DES was administered as a salvage therapy either after first-line hormonal therapy (LH-RH and bicalutamide) or after a trial of chemotherapy. The medical records of these patients were located and thoroughly reviewed, collecting the relevant information into a dedicated database. All patients had CRPC, having progressed biochemically and/or clinically while under treatment with an LH-RH analog prior to DES therapy. Information concerning the length of treatment and the biochemical response to LH-RH analogs, based on serum prostate-specific antigen (PSA) measurements, was collected and analyzed. Additional data collected included patient age at diagnosis, Gleason score, presence of distant metastases at diagnosis, previous chemotherapy, concomitant medications, adverse effects attributable to DES and the administered dose of DES. Only patients with sufficient information concerning their biochemical response to both LH-RH analogs and DES were included in the correlation analysis.

DES formulation. DES capsules were prepared by a local Jerusalem pharmacy (Alba Pharmacy) by mixing dry DES powder obtained from Sigma-Aldrich and other chemical suppliers with magnesium oxide as bulk excipient and dispensed in soft gel capsules containing $1 \mathrm{mg}$ DES.

Study endpoints. The clinical and biochemical course of the disease was closely followed by periodic clinic visits and serum PSA measurements. The end of the follow-up period was defined as July 1, 2011.

The primary endpoint was time to disease progression (TDP) in the LH-RH and DES treatment phases and intrapatient correlation analysis of TDP. TDP was calculated as the period of time between initiation of LH-RH therapy or DES therapy and one of the following: biochemical disease progression, switching to a different treatment, patient death or end of follow-up. Biochemical disease progression (PSA progression) was defined in accordance with the Prostate Cancer Clinical Trials Working Group (PCWG2) as 'a $25 \%$ or greater increase and an absolute increase of $2 \mathrm{ng} / \mathrm{ml}$ or more from the nadir which is confirmed by a second value obtained 3 or more weeks later' (4).

Secondary endpoints included analysis of other possible predictive factors of TDP (Gleason grade and PSA response), survival time and cessation of DES therapy due to side-effects or for any reason other than disease progression.

Statistical methods. All statistical analyses were performed using 'IBM SPSS Statistics version 20' and 'Microsoft Excel 2010'. Univariate analysis and regression analysis using Pearson's product-moment and Spearman's rank correlation coefficients were undertaken to evaluate factors predictive for the response to DES in those patients. Median time to disease progression (TDP) and overall survival were obtained using 
Table I. Patient characteristics.

\begin{tabular}{lc}
\hline Characteristics & Patient data \\
\hline Age at diagnosis (years) & $66(49-88)$ \\
Median (range) & \\
Months between diagnosis and DES initiation & $50(5-180)$ \\
Median (range) & \\
Distant metastases present at diagnosis & $25(58)$ \\
Yes (\%) & $18(42)$ \\
No (\%) & $12(28 \%)$ \\
Previous chemotherapy & $31(72 \%)$ \\
Yes (\%) & \\
No (\%) & $47(4.9-6,255)$ \\
Serum PSA at diagnosis (ng/ml) & \\
Median (range) & $18(3.0-1.580)$ \\
Serum PSA at DES initiation (ng/ml) & \\
Median (range) & $6(14)$ \\
Gleason score sum, $(\%)$ & $9(21)$ \\
$4-6$ & $14(32.5)$ \\
7 & $14(32.5)$ \\
8-10 & \\
\hline &
\end{tabular}

the Kaplan-Meier method. The log-rank test was used to compare the curves. Patients who were lost to follow-up were censored at the end of the follow-up period.

Baseline PSA was defined as the last serum PSA measurement before initiation of DES therapy. The PSA nadir was defined as the lowest value of serum PSA that was measured while the patient was receiving DES. PSA response was calculated as the decrement in serum PSA from the baseline to the nadir expressed as a percentage of the baseline serum PSA.

Toxicities were considered secondary to treatment if they occurred while the patient was receiving DES or up to 2 months after treatment termination.

\section{Results}

Patient characteristics. Forty-six patient records were located; 3 did not have sufficient follow-up information, leaving a study sample consisting of 43 patients. Out of the 43 patients, 38 $(88 \%)$ had sufficient follow-up information and were eligible for correlation analysis. The patient characteristics are summarized in Table I. Patients were diagnosed at a median age of 66 years and were treated with LH-RH analogs for a median period of 34 months. Following disease progression, based on biochemical (PSA rise only) or biochemical and clinical progression (PSA rise and new or progressive metastases), patients started receiving DES after a median period of 50 months from the time of diagnosis. Twenty-five (58\%) patients had evidence of distant metastases at the time of diagnosis. Twelve (28\%) patients had received some form of chemotherapy prior to DES. The median serum PSA was $47 \mathrm{ng} / \mathrm{ml}$ at the time of diagnosis and $18 \mathrm{ng} / \mathrm{ml}$ at start of DES therapy.
Although the pathological diagnosis of adenocarcinoma of the prostate was confirmed in all 43 patients, Gleason scoring was available in only 29 patients. Six patients had a low score (between 4 and 6), 9 patients had an intermediate score (7) and 14 patients had a high score (between 8 and 10).

Therapy. The planned dose of DES was $3 \mathrm{mg} /$ day based on our previous experience (17), but the actual daily dose of DES ranged from 1 to $4 \mathrm{mg} /$ day. Two patients $(5 \%)$ received $1 \mathrm{mg} /$ day, 12 patients $(30 \%)$ received $2 \mathrm{mg}$ /day, 25 patients $(62.5 \%)$ received $3 \mathrm{mg} /$ day and one patient $(2.5 \%)$ received $4 \mathrm{mg}$ /day $(\mathrm{N}=40)$. The mean daily dose was $2.6 \mathrm{mg} /$ day. The median duration of treatment was 20.8 (range, 0.33-136.5) months. All patients continued LH-RH treatment. However, in some of the long-term responding patients, $\mathrm{LH}-\mathrm{RH}$ analogs were eventually discontinued and testosterone levels still remained at castrate levels.

Concomitant therapy was mainly antiplatelet or anticoagulation therapy as primary prophylaxis for venous thromboembolic events (VTEs) and bisphosphonates. While all patients were advised to take anticoagulants, only 36 patients were confirmed to receive anticoagulants: 30 patients (75\%) received acetylsalicylic acid, 6 patients $(15 \%)$ received warfarin and 1 patient $(2.5 \%)$ received low-molecular-weight heparin (LMWH). Twenty-two (55\%) patients received bisphosphonates. Nearly all patients received prophylactic breast irradiation for prevention of gynecomastia.

PSA response. A biochemical PSA response ( $\geq 50 \%$ decrease) to DES therapy was achieved in $24 / 38(63 \%)$ patients and any PSA decline was observed in $34 / 38$ (89\%) patients. The median maximal change in serum PSA under DES therapy was $-66.5 \%$ (range, -100 to $309 \%$ ). Fig. 1 shows a waterfall plot of the maximal changes in serum PSA response during LH-RH (Fig. 1A) and DES (Fig. 1B) treatment. Based on the change in PSA, the vast majority of patients responded favorably to both treatments.

Time to disease progression (TDP). The Kaplan-Meier curves of the TDP under LH-RH analogs and under DES therapy are shown in Fig. 2. The median TDP was 7.1 (95\% CI, 5.4-8.8) months under DES therapy, and 20.4 (95\% CI, 13.627.3) months under LH-RH analogs. The TDP under DES was significantly shorter $(\mathrm{P}=0.003)$ than that under $\mathrm{LH}-\mathrm{RH}$ analogs.

The median TDP in patients treated with DES was similar for patients with metastatic and localized disease at diagnosis; 8.2 (95\% CI, 5.5-11) months and 7.1 (95\% CI, 6.7-7.5) months, respectively $(\mathrm{P}=0.74)$. Of note, 12 patients $(31 \%)$ had durable responses ( $>12$ months) to DES.

Predictors of response duration. Correlation analyses demonstrated that TDP under LH-RH analogs was not predictive of the TDP under subsequent DES treatment (Pearson $\mathrm{P}=0.518$ and Spearman $\mathrm{P}=0.288$ ). This is illustrated graphically in Fig. 3.

Significant univariate correlates for TDP under DES included a high baseline serum PSA prior to initiation of DES which was associated with shorter TDP under DES (Spearman rho $=-0.401 ; \mathrm{P}=0.017$ ), and the magnitudes of maximal serum 

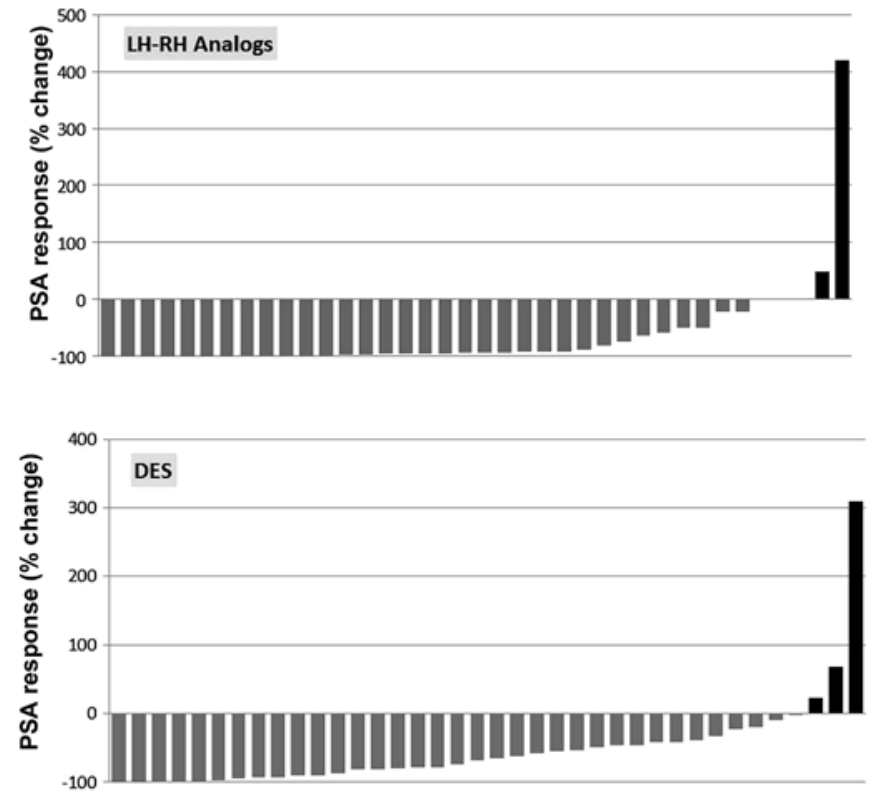

Figure 1. Waterfall plot of maximal PSA response to (A) LH-RH analogs and (B) DES. Each bar represents a different patient. PSA, prostate-specific antigen; LH-RH, luteinizing hormone-releasing hormone; DES, diethylstilbestrol.

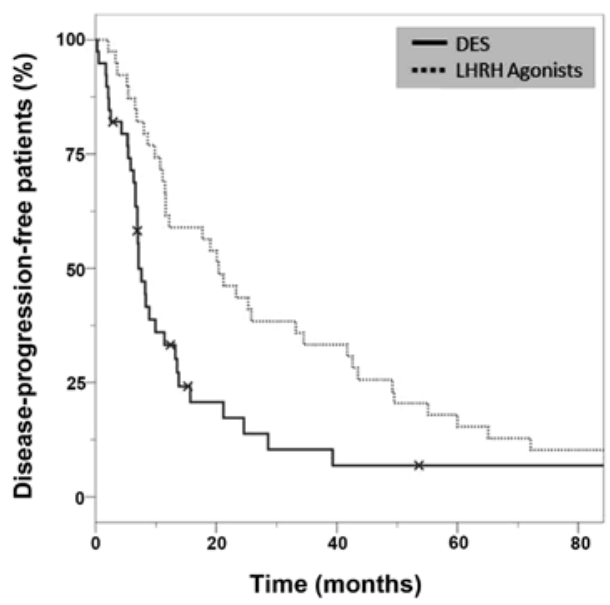

Figure 2. Kaplan-Meier curve of TDP under DES and LH-RH analogs. TDP, time to disease progression; DES, diethylstilbestrol; LH-RH, luteinizing hormone-releasing hormone.

PSA response (\% decline from baseline to nadir) to LH-RH (Spearman rho $=0.334 ; \mathrm{P}=0.05$ ) and to DES (Spearman rho $=0.506 ; \mathrm{P}=0.002$ ) which were correlated with longer TDP under DES.

Among the 29 patients with Gleason scores at the time of diagnosis, univariate analysis showed that TDP was inversely proportional to Gleason scores for first line therapy with LH-RH. In contrast, there was no significant correlation between Gleason score and the TDP for salvage therapy with DES [Spearman correlation analysis $(\mathrm{P}=0.926)]$. This is illustrated in Fig. 4.

Survival. Fifty-five percent of the patients were deceased at the end of the follow-up period. The median overall survival after

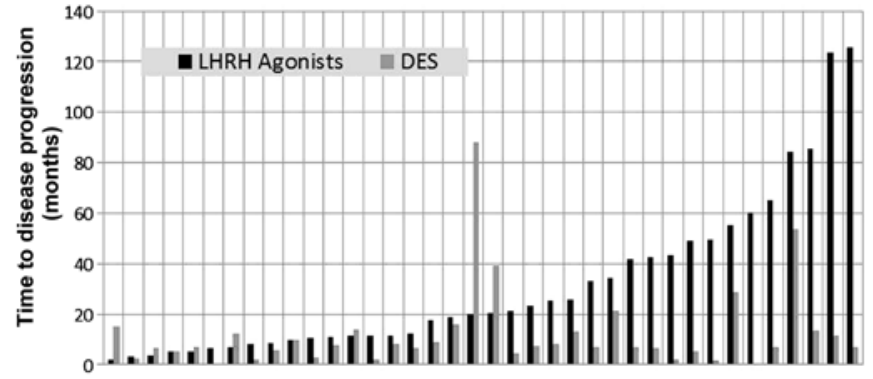

Figure 3. TDP under LH-RH analogs and DES. Each set of 2 bars represents a different patient. TDP, time to disease progression; $\mathrm{LH}-\mathrm{RH}$, luteinizing hormone-releasing hormone; DES, diethylstilbestrol.

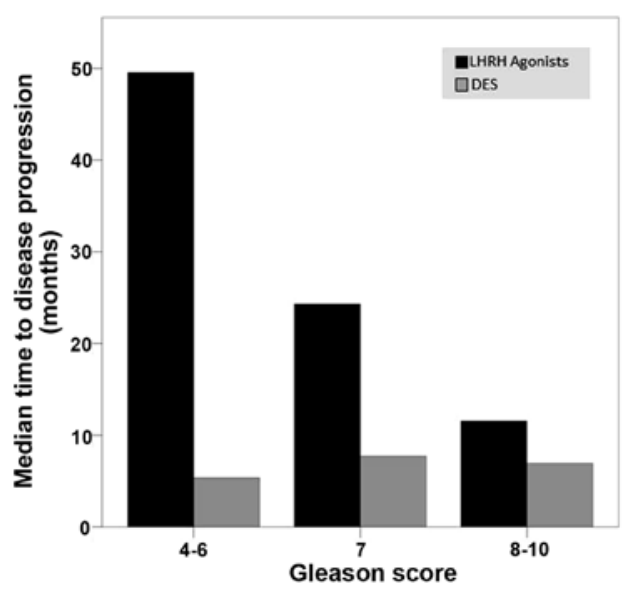

Figure 4. Median TDP under DES and LH-RH analogs for patients with low (4-6), intermediate (7) and high (8-10) Gleason scores at diagnosis. TDP, time to disease progression; DES, diethylstilbestrol; LH-RH, luteinizing hormone-releasing hormone.

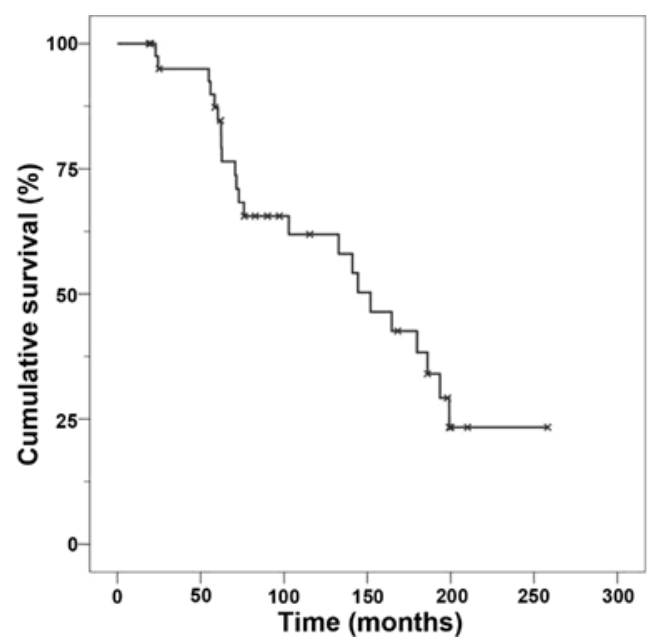

Figure 5. Kaplan-Meier plot of overall survival after diagnosis.

diagnosis was 151.8 (95\% CI, 115-188) months (Fig. 5). The median survival after initiation of DES therapy was 57.1 (95\% CI, 30-84) months (Fig. 6). As expected, median survival after diagnosis for patients with localized disease (186 months) was significantly longer than for patients with metastatic disease (103 months) $(\mathrm{P}=0.029)$. 
Table II. Comparison of studies using DES in CRPC.

\begin{tabular}{|c|c|c|c|c|c|c|c|}
\hline Authors (ref.) & Year & $\begin{array}{l}\text { No. of } \\
\text { patients }\end{array}$ & $\begin{array}{c}\text { Therapy } \\
\text { (daily dose) }\end{array}$ & $\begin{array}{l}\text { PSA }(\geq 50 \%) \\
\text { response, } \%\end{array}$ & $\begin{array}{c}\text { Median } \\
\text { TDP } \\
\text { (months) }\end{array}$ & $\begin{array}{l}\text { Median } \\
\text { survival } \\
\text { (months) }\end{array}$ & $\begin{array}{c}\text { Thromboembolic } \\
\text { events, } \%\end{array}$ \\
\hline Grenader et al (present study) & 2013 & 43 & DES (2-3 mg) & 63 & 7.1 & 57 & 9 \\
\hline Clemons et al (18) & 2013 & 58 & DES (1 mg) & 39 & $4.0^{\mathrm{a}}$ & na & 3 \\
\hline Wilkins et al (3) & 2012 & 231 & DES (1mg) & 29 & 4.6 & 9.3 & 10 \\
\hline Shamash et al (19) & 2010 & 145 & $\begin{array}{l}\text { DES }(1 \mathrm{mg})+ \\
\text { Dexamethasone }\end{array}$ & 68 & 8.0 & 18.3 & 11 \\
\hline Manikandan et al (20) & 2005 & 26 & DES (1 mg) & 27 & 9.0 & $>24^{\mathrm{b}}$ & 8 \\
\hline Rosenbaum et al (17) & 2000 & 18 & DES (3 mg) & 66 & 7.5 & na & 0 \\
\hline Orlando et al (21) & 2000 & 38 & $\begin{array}{l}\text { Fosfestrol } \\
\text { (DES pro-drug) }\end{array}$ & 58 & 7.0 & 12 & 8 \\
\hline Smith et al (22) & 1998 & 21 & DES (1 mg) & 43 & na & $>24^{\mathrm{c}}$ & 5 \\
\hline
\end{tabular}

na, not available. ${ }^{a}$ TDP in responding patients, 7.5 months; ${ }^{b} 54 \%$ alive at median follow-up of 24 months; ${ }^{\mathrm{c}} 63 \%$ estimated survival rate at 24 months. DES, diethylstilbestrol; CRPC, castration-resistant prostate cancer; PSA, prostate-specific antigen; TDP, time to disease progression.

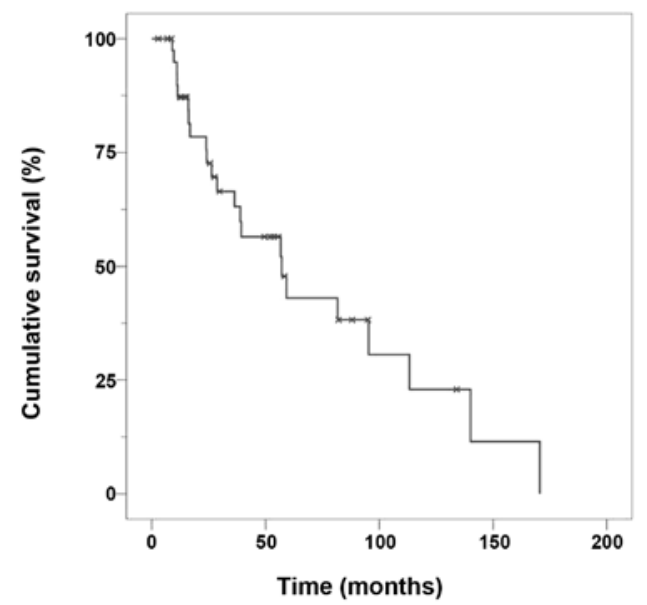

Figure 6. Kaplan-Meier plot of overall survival after initiation of DES. DES, diethylstilbestrol.

Toxicity. The DES treatment was generally well-tolerated by the patients. There was not a single case of mortality during DES therapy or as a result of thromboembolic complications during the follow-up period.

A total of 4 patients (9.3\%) developed thromboembolic complications. Of these, $1(2.3 \%)$ had deep venous thromboembolism (DVT), 2 (4.7\%) had a pulmonary embolism (PE) and 1 patient $(2.3 \%)$ had both. Two of the 4 patients who suffered a thromboembolic complication were not receiving prophylactic anticoagulation. There were 1 and 2 thromboembolic events for patients who received 2 and $3 \mathrm{mg}$ of DES, respectively.

One patient (2.4\%) experienced a cerebrovascular accident. No myocardial infarctions or other ischemic complications were reported.

The following other toxic effects were observed: peripheral edema in 11 patients (25.6\%); CHF exacerbation in 1 patient $(2.4 \%)$; gynecomastia in 6 patients $(14 \%)$; mastodynia in 2 patients $(4.7 \%)$; nausea in 5 patients $(11.6 \%)$; vomiting in 3 patients (7\%). No other significant drug-related adverse effects were reported.

\section{Discussion}

DES is a fascinating medication. Despite a history of over 5 decades of experience, the exact mechanism of action of DES among patients with castration-resistant prostate cancer remains unknown. Previous studies have suggested several possibilities that may account for its antitumor effect. Suppression of the hypothalamic-testicular axis, thus reducing serum testosterone to castrate levels (1), and suppression of adrenal androgens such as dehydroandrostendione and dehydroepiandrosterone sulfate (14) are possible mechanism of action. In addition to these hormonally mediated effects, DES may interfere with the cell cycle and induce apoptosis of androgen-dependent and -independent prostate cancer cell lines (23). Various mechanisms have been proposed including inhibition of microtubule assembly (24), inhibition of telomerase (25) and inhibition of DNA synthesis (26). It was also demonstrated by Schulz et al (27) that DES interferes with cellular energy metabolism by inhibiting the bc- 1 complex in the mitochondrial respiratory chain. Finally, there is evidence that DES, as in the case of 2-methoxy-estradiol, may directly inhibit angiogenesis (26).

Based on this small cohort of patients, DES appears to be highly beneficial to patients with CRPC, with a median TDP of 7.1 months, durable responses ( $>12$ months) in $31 \%$ patients, and a biochemical PSA response rate of $63 \%$. DES was effective in pre-, as well as post-chemotherapy-treated patients with CRPC.

We found a significant correlation between the magnitude of serum PSA response to LH-RH agonists, the magnitude of serum PSA response to DES and the TDP under DES. These findings suggest that the magnitude of serum PSA response to LH-RH agonists can perhaps be used to predict the magnitude and duration of response to DES in patients with CRPC. 
However, there was no correlation between the TDP under LH-RH analogs and DES. Notably, the Gleason grade was highly predictive of the TDP under LH-RH analogs but not so of the TDP under DES therapy, suggesting important differences in the anticancer mechanism of action of both agents. In the study of Shamash et al (19), a PSA decrease $>50 \%$ within 1 month of treatment with DES and corticosteroids was found to predict a favourable prognosis with median TDP greater than 1 year and median survival greater than 2 years.

A comparison of studies that used 1-3 mg DES in CRPC points to a relatively high PSA response rate (27-66\%) and roughly similar median TDP (4-9 months) across all studies (Table II). These findings add to the accumulated evidence that there is substantial potential gain from a therapeutic trial with DES even among patients who have had a very short response to prior LHRH agonist therapy. An apparently higher response rate in patient groups receiving 2-3 $\mathrm{mg}$ as opposed to $1 \mathrm{mg}$ DES can be noted (Table II).

A review by Bosset et al (12) reports that thromboembolic events occur in $~ 5 \%$ of patients receiving DES with thromboprophylaxis. The rate of thromboembolic complications in the present study was $9 \%$, similar to that observed in previous studies (Table II). Overall, DES was found to be a relatively safe treatment option, when thromboprophylaxis measures are rigorously observed.

Proactive management of side-effects and patient education are vital. Prophylactic breast radiotherapy can reduce the incidence of gynecomastia. Patients should be strongly counseled to take prophylactic antiplatelet or anticoagulation agents to prevent potential venous thromboembolism. No prospective controlled studies have been performed to determine which drug is most appropriate to prevent thromboembolic events under DES treatment. Further research is required to standardize prophylactic treatment and improve the safety profile of DES.

The present study has a number of limitations. The database from which the patients were retrieved was incomplete and some of the patients were identified by interviewing oncologists who have used DES in our medical facility. Due to the small cohort size, the study was probably underpowered to detect statistical differences in some of the analyses performed, and multivariate analysis could not be performed.

In the last few years, the number of medicines approved for the treatment of CRPC has increased dramatically, and includes the hormonal agents abiraterone (14) and MDV3100 (enzalutamide), the cytotoxic agent cabazitaxel (28), an adoptive cellular immunotherapy Sipuleucel-T (29) and alpharadine, a bone-targeted radionuclide Radium-223 (30). In addition, promising early clinical data in CRPC have been obtained with cabozantinib, a tyrosine kinase inhibitor.

Cancer treatment costs in CRPC have increased dramatically in the last decade with the regular introduction of novel and expensive systemic therapies. For example, abiraterone was initially rejected by NICE for use in the UK and Wales due to its high price. Guidelines have been reissued, after the manufacturer agreed to a reduced price of $£ 2,930$ for a month's supply. The cost of abiraterone per quality adjusted life year (QALY) gained was $£ 63,200$ and $£ 46,800$ per QALY, pre- and post-discount, respectively (31). In the pharmaco-economic context, the much lower cost of DES offers a conspicuous advantage over some of the new CRPC therapies.

In conclusion, DES is an effective agent for treatment of CRPC, with an acceptable safety profile when administered together with antiplatelet or anticoagulation agents. Although responses are significantly shorter-lasting than the relatively long responses to first-line treatment with LH-RH analogs, DES stands out as an important and inexpensive therapeutic option for patients with CRPC. DES is especially important in patients who are not candidates for chemotherapy or as a therapeutic option preceding chemotherapy in patients with CRPC. Thromboprophylaxis is critical for the safe use of DES. The exact mechanism of action of DES in prostate cancer remains unclear and is most likely multifactorial. Further research is required to understand its role in CRPC and to establish its place in the treatment-sequence algorithm.

\section{Acknowledgements}

The present study was supported in part by a grant of the Israel Cancer Association to Y.P. The present study was conducted, in part, for the requirement of the Hebrew University of Jerusalem, Medical School for awarding the degree of 'Doctor of Medicine' to Y.P.

\section{References}

1. Siegel R, DeSantis C, Virgo K, Stein K, Mariotto A, Smith T, Cooper D, et al: Cancer treatment and survivorship statistics. CA Cancer J Clin 62: 220-241, 2012.

2. Huang $\mathrm{X}$, Chau $\mathrm{CH}$ and Figg WD: Challenges to improved therapeutics for metastatic castrate resistant prostate cancer: from recent successes and failures. J Hematol Oncol 5: 35, 2012. doi: 10.1186/1756-8722-5-35.

3. Wilkins A, Shahidi M, Parker C, Gunapala R, Thomas K, Huddart R, Horwich A, et al: Diethylstilbestrol in castrationresistant prostate cancer. BJU Int 110: E727-E735, 2012.

4. Scher HI, Halabi S, Tannock I, Morris M, Sternberg CN, Carducci M, et al: Design and end points of clinical trials for patients with progressive prostate cancer and castrate levels of testosterone: recommendations of the Prostate Cancer Clinical Trials Working Group. J Clin Oncol 26: 1148-1159, 2008.

5. Petrylak DP, Tangen CM, Hussain MH, Lara PN, Jones JA, Taplin ME, Burch PA, et al: Docetaxel and estramustine compared with mitoxantrone and prednisone for advanced refractory prostate cancer. New Eng1 J Med 351: 1513-1520, 2004.

6. Tannock, IF, De Wit R, Berry WR, Horti J, Pluzanska A, Chi KN, Oudard S, et al: Docetaxel plus prednisone or mitoxantrone plus prednisone for advanced prostate cancer. New Engl J Med 351: 1502-1512, 2004.

7. Locke JA, Guns ES, Lubik AA, Adomat HH, Hendy SC, Wood CA, Ettinger SL, Gleave ME and Nelson CC: Androgen levels increase by intratumoral de novo steroidogenesis during progression of castration-resistant prostate cancer. Cancer Res 68: 6407-6415, 2008.

8. Montgomery RB, Mostaghel EA, Vessella R, Hess DL, Kalhorn TF, Higano CS, True LD and Nelson PS: Maintenance of intratumoral androgens in metastatic prostate cancer: a mechanism for castration-resistant tumor growth. Cancer Res 68: 4447-4454, 2008.

9. Aggarwal R, Weinberg V, Small EJ, Oh W, Rushakoff R and Ryan CJ: The mechanism of action of estrogen in castrationresistant prostate cancer: clues from hormone levels. Clin Genitourin Cancer 7: E71-E76, 2009.

10. Huggis $C$ and Hodges CV: Studies on prostatic cancer. I. The effects of castration, of estrogen and of androgen injection on serum phosphatases in metastatic carcinoma of the prostate. Cancer Res 1: 293-297, 1941. 
11. Veterans Administration Cooperative Urological Research Group: Carcinoma of the prostate: treatment comparison. J Urol 98: 516-522, 1967

12. Bosset PO, Albiges L, Seisen T, De la Motte Rouge T, Phé V, Bitker MO and Rouprêt M: Current role of diethylstilbestrol in the management of advanced prostate cancer. BJU Int 110: E826-E829, 2012.

13. Malkowicz SB: The role of diethylstilbestrol in the treatment of prostate cancer. Urology 58: 108-113, 2001.

14. Kitahara S, Yoshida K, Ishizaka K, Kageyama Y, Kawakami S, Tsujii $\mathrm{T}$ and Oshima H: Stronger suppression of serum testosterone and FSH levels by a synthetic estrogen than by castration or an LH-RH agonist. Endocr J 44: 527-532, 1997.

15. Robinson MR, Smith PH, Richards B, Newling DW, De Pauw M and Sylvester R: The final analysis of the EORTC GenitoUrinary Tract Cancer Co-Operative Group phase III clinical trial (protocol 30805) comparing orchidectomy, orchidectomy plus cyproterone acetate and low dose stilboestrol in the management of metastatic carcinoma of the prostate. Eur Urol 28: 273-283, 1985.

16. Montgomery B , Nelson PS , Vessella R , Kalhorn T, Hess D and Corey E: Estradiol suppresses tissue androgens and prostate cancer growth in castration resistant prostate cancer. BMC Cancer 10: 244, 2010.

17. Rosenbaum E, Wygoda M, Gips M, Hubert A, Tochner Z and Gabizon A: Diethylstilbestrol is an active agent in prostatic cancer patients after failure to complete androgen blockade. Proc J Clin Oncol 19: 349a, 2000.

18. Clemons J, Glodé LM, Gao D and Flaig TW: Low-dose diethylstilbestrol for the treatment of advanced prostate cancer. Urol Oncol 31: 198-204, 2013.

19. Shamash J, Stebbing J, Sweeney C, Sonpavde G, Harland S, Dawkins $\mathrm{G}$, Brock $\mathrm{C}$, et al: A validated prognostic index predicting response to dexamethasone and diethylstilbestrol in castrate-resistant prostate cancer. Cancer 116: 3595-3602, 2010.

20. Manikandan R, Srirangam SJ, Pearson E, Brown SC, O'Reilly P and Collins GN: Diethylstilboestrol versus bicalutamide in hormone refractory prostate carcinoma: a prospective randomized trial. Urol Int 75: 217-221, 2005.

21. Orlando M, Chacón M, Salum G and Chacón DR: Low-dose continuous oral fosfestrol is highly active in 'hormone-refractory' prostate cancer. Ann Oncol 11: 177-181, 2000.
22. Smith DC, Redman BG, Flaherty LE, Li L, Strawderman M and Pienta KJ: A phase II trial of oral diethylstilbesterol as a secondline hormonal agent in advanced prostate cancer. Urology 52: 257-260, 1998

23. Robertson CN, Roberson KM, Padilla GM, O'Brien ET, Cook JM, Kim CS and Fine RL: Induction of apoptosis by diethylstilbestrol in hormone-insensitive prostate cancer cells. J Natl Cancer Inst 88: 908-917, 1996

24. Hartley-Asp B, Deinum J and Wallin M: Diethylstilbestrol induces metaphase arrest and inhibits microtubule assembly. Mutat Res 143: 231-235, 1985.

25. Geier R, Adler S, Rashid G and Klein A: The synthetic estrogen diethylstilbestrol (DES) inhibits the telomerase activity and gene expression of prostate cancer cells. Prostate 70: 1307-1312, 2010.

26. Ho SM: Estrogens and anti-estrogens: key mediators of prostate carcinogenesis and new therapeutic candidates. J Cell Biochem 91: 491-503, 2004.

27. Schulz P, Link TA, Chaudhuri L and Fittler F: Role of the mitochondrial bc1-complex in the cytotoxic action of diethylstilbestrol-diphosphate toward prostatic carcinoma cells. Cancer Res 50: 5008-5012, 1990.

28. de Bono JS, Oudard S, Ozguroglu M, Hansen S, Machiels JP, Kocak I, Gravis G, et al: Prednisone plus cabazitaxel or mitoxantrone for metastatic castration-resistant prostate cancer progressing after docetaxel treatment: a randomised open-label trial. Lancet 376: 1147-1154, 2010.

29. Kantoff PW, Higano CS, Shore ND, Berger ER, Small EJ, Penson DF, Redfern CH, Ferrari AC, Dreicer R, Sims RB, $\mathrm{Xu}$ Y, Frohlich MW and Schellhammer PF; IMPACT Study Investigators: Sipuleucel-T immunotherapy for castrationresistant prostate cancer. New Engl J Med 363: 411-422, 2010.

30. Gerger A, Langsenlehner U, Kapp K and Langsenlehner T: Overall survival benefit and safety profile of radium-223 chloride, a first-in-class alpha-pharmaceutical: results from a phase III randomized trial (ALSYMPCA) in patients with castrationresistant prostate cancer (CRPC) with bone metastases. J Clin Oncol 30 (Suppl 5): 8, 2012.

31. Kmietowicz Z: NICE recommends abiraterone for prostate cancer after manufacturer reduces price. BMJ 344: e3520, 2012. 